\title{
DATING GODS: RADIOCARBON DATES FROM THE SANCTUARY OF ZEUS ON MT. LYKAION (ARCADIA, GREECE)
}

\author{
Britt M Starkovich $^{1,2}$ • Gregory W L Hodgins ${ }^{2,3} \bullet$ Mary E Voyatzis $^{2} \bullet$ David Gilman Romano $^{2}$
}

ABSTRACT. This paper reports the results of the application of a calcined bone dating protocol to samples collected from the Sanctuary of Zeus on Mt. Lykaion in southern Greece. The site is a mountaintop ash altar rich in anthropogenic sediments, burned bone, and artifacts offered to the god Zeus. Experiments involving time series hydrolysis measurements were conducted on calcined bones from stratified layers throughout the sequence to determine if any of the samples underwent surface contamination from carbonate exchange with the surrounding sedimentary matrix. It was determined that such exchange was unlikely, but samples were acid-etched before pretreatment as a precautionary measure. Paired samples of seeds, charcoal, and calcined animal bone collected from a sediment column in the altar demonstrate the effectiveness of the calcined bone dating technique in this context. The results of dating indicate that the altar was in use from the Mycenaean period through the late Classical period, though samples were not collected from the upper levels of the site due to possible mixing of surface sediments. Fourier transform infrared spectroscopy (FTIR) measurements were taken and crystallinity index values calculated, confirming that the bone samples are indeed calcined. The results presented here correspond with literary accounts of ritual animal sacrifice from historical texts from the 8th century BCE, including the Homeric epics.

\section{INTRODUCTION}

Radiocarbon dating bone has historically proven to be difficult in the absence of preserved organic collagen. Unfortunately, collagen is extremely vulnerable to degradation, particularly when burned. It has become increasingly apparent in recent years that ${ }^{14} \mathrm{C}$ dating structural carbonates is possible when bones are burned to the point of being calcined (whitened). Multiple calcined bone dating protocols introduced in the last decade yield reliable results when compared with ${ }^{14} \mathrm{C}$ dates from classes of artifacts found in contemporaneous contexts (e.g. Lanting et al. 2001; De Mulder et al. 2007; Olsen et al. 2008; Van Strydonck et al. 2009). In this study, a dating protocol inspired by the previously mentioned authors is applied to an archaeological sequence of calcined bones. The faunal remains from the Sanctuary of Zeus on Mt. Lykaion, in southern Greece, provide an ideal test case for the calcined bone dating method. The depositional context is a mountaintop ash altar with over a meter of anthropogenic sediments rich in calcined bone, ash, ceramics, and botanical remains. Diagnostic ceramics date the material found in the ash altar from the Final Neolithic period through the Late Classical or Hellenistic period (about 6000 to $2300 \mathrm{yr}$ ago). This paper presents a series of ${ }^{14} \mathrm{C}$ dates taken from a sediment column in the deepest part of the altar. The goals of the study are to: 1) test the validity of the calcined bone dating method in this particular context; 2) determine the stratigraphic integrity and possible postdepositional movement of small artifacts in the altar deposits; and 3) establish the timespan during which the altar was in use. This study differs from previous works in that the bones are animal rather than human, and they come exclusively from an open-air surface context, as opposed to rapidly interred burials.

\section{BACKGROUND}

In landmark studies by Lanting and colleagues (Lanting and Brindley 1998; Lanting et al. 2001), it was established that calcined bone has the potential to yield reliable ${ }^{14} \mathrm{C}$ dates. Over a decade of research now confirms the efficacy of this technique, and the reproducibility of calcined bone dates

\footnotetext{
${ }^{1}$ Institut für Naturwissenschaftliche Archäologie, Universität Tübingen, Germany. Corresponding author

Email: britt.starkovich@uni-tuebingen.de.

${ }^{2}$ School of Anthropology, University of Arizona, Tucson, Arizona, USA.

${ }^{3}$ NSF-Arizona AMS Laboratory, University of Arizona, Tucson, Arizona, USA.
} 


\section{B M Starkovich et al.}

between different ${ }^{14} \mathrm{C}$ facilities (Naysmith et al. 2007). The method relies on the dating of inorganic structural carbonate ("bioapatite") as opposed to organic collagen that is typically used when dating unburned bone. During burning, bone collagen is destroyed at fairly low temperatures (200$250{ }^{\circ} \mathrm{C}$ ), while higher-temperature burning $\left(>600^{\circ} \mathrm{C}\right)$ causes bioapatite to recrystallize into larger, more stable crystals (Shipman et al. 1984; Quarta et al. 2012). These crystals form a more compact structure than that of unburned bone, and are less susceptible to contamination from carbonate ion exchange with the surrounding sediments, effectively forming a barrier between the surface and interior of the bone (Van Strydonck et al. 2005, 2009). Van Strydonck et al. (2009) showed that under certain environmental conditions, small amounts of surface ion exchange occurs, and recommend acid-leaching the exterior of calcined bone samples before pretreatment to remove possible contaminants.

The most recent generation of calcined bone dating research experimentally tests possibilities for the origin of carbon in calcined bone, beyond atmospheric carbon, specifically examining collagen (Zazzo et al. 2009) and fuel sources (Hüls et al. 2010; Van Strydonck et al. 2010). Zazzo et al. (2009) determine that organic collagen is an unlikely source for the carbon in calcined bioapatite because the organic portion of bone gets destroyed at a low temperature, long before recrystallization. Hüls et al. (2010) and Van Strydonck et al. (2010) seek to test the hypothesis that if "old wood" is combusted during cremation, old carbon is incorporated into bioapatite as it recrystallizes, causing the bone to reflect the date of the wood, as opposed to the date of the cremation. The authors find that using closed furnaces for combustion in laboratory settings can cause "old wood" carbon to recrystalize into bioapatite, but conclude that this probably only occurs under very specific circumstances, and is extremely rare archaeologically (Van Strydonck et al. 2010, but see Olsen et al. 2012 for an archaeological example of this phenomenon). Incidentally, the top of Mt. Lykaion is harshly exposed and typically windy, so it is unlikely that large amounts of old carbon would incorporate into calcined bone, even if old wood was used as fuel.

Calcined bone dating has successfully been applied to the archaeological record, primarily in the context of human cremations in northern Europe (e.g. Sheridan 2003; De Mulder et al. 2007, 2009; Olsen et al. 2008, 2011; Van Strydonck et al. 2009, but see Zazzo et al. 2009 for dating calcined animal bones in the Near East and Africa). Due to the depositional environment and suite of available materials for dating, including ample calcined bone, the Sanctuary of Zeus is an excellent location for applying the calcined bone dating technique.

The Sanctuary of Zeus on Mt. Lykaion is located in the mountains of Arcadia, near the modern city of Megalopolis in the central Peloponnese. The site includes a Lower Sanctuary, used primarily during Archaic, Classical, and Hellenistic times, and an Upper Sanctuary, comprised of a temenos (sacred precinct) and a mountaintop ash altar. The altar lies at $1382 \mathrm{~m}$ above sea level, covering the entire top of the mountain $\left(520 \mathrm{~m}^{2}\right)$. Initial archaeological excavations took place at the site at the turn of the 20th century (Kontopoulous 1898; Kourouniotis 1904). Recent investigations at the altar began with a computerized architectural and topographical study of the site in 1996 (Romano 2005), which subsequently led to excavations from 2007 to 2010 by the University of Arizona, the University of Pennsylvania Museum, and the 39th Ephoria of Prehistoric and Classical Antiquities in Tripolis (Romano and Voyatzis, forthcoming). Two trenches were excavated on the altar, one northwest to southwest across the top (Trench Z, $2 \times 14 \mathrm{~m}$ ) and one along the southern edge of the mountaintop (Trench ZZ, $2 \times 6 \mathrm{~m}$ ). All samples discussed in this study were collected from Trench Z.

Sediments on the altar are up to a meter and a half thick, and are nearly entirely anthropogenic in origin (Mentzer, forthcoming; Mentzer et al., forthcoming). This unique depositional environment is 
${ }^{14}$ C Dates from the Sanctuary of Zeus on Mt. Lykaion

extremely rich in fragments of heavily burned sheep or goat femurs, patellas, and caudal (tail) vertebrae (Starkovich, forthcoming), offered to the god Zeus as part of a ritual called thysia (or ritual sacrifice). During this practice, which is referenced in both Homer's Iliad (2:420-430) and Odyssey (3:418-463), large animals are led to an altar, splashed with barley and water, sacrificed, and dismembered. Certain limb bones, such as the femur, were defleshed, wrapped in fat, and burned as an offering to the gods. Tails were often burned for divining or oracular purposes (Ekroth 2007, 2008, 2009). In addition to hundreds of thousands of calcined bone fragments, thousands of pieces of ceramic vessels, terracotta figurines, bronze and silver coins, miniature bronze tripod cauldrons, barley seeds, olive pits, and roof tiles were recovered from the altar (Romano and Voyatzis, forthcoming). Micromorphological analyses indicate that the surrounding sedimentary matrix is comprised almost exclusively of bone, ash, and fat-derived char, down to the sand and silt-sized particles (see Mentzer, forthcoming; Mentzer et al., forthcoming, for detailed methodology and results). Ceramic evidence indicates that the altar was in use from the Final Neolithic through the 4th century BCE, with substantial use during the Mycenaean and later periods (Romano and Voyatzis, forthcoming). Altars of various types are known throughout Greece, but evidence for burnt animal sacrifice in the Bronze Age, as is apparent on Mt. Lykaion, is rare.

\section{METHODS}

\section{Sample Selection}

In order to test the validity of the calcined bone dating method at the ash altar on Mt. Lykaion, a series of $72{ }^{14} \mathrm{C}$ dates from a $25 \times 25 \times 50 \mathrm{~cm}$ sediment column was taken from the deepest part of the site (Figure 1). Due to the possible mixing of sediments at the surface of the site, sample collection began about $50 \mathrm{~cm}$ below the surface. The column was excavated in $5-\mathrm{cm}$ cuts, and 60 paired samples of charcoal, seeds, and calcined bones were collected from every layer. Calcined bone samples were collected during excavation and were double wrapped in aluminum foil before storage in plastic bags. Charcoal and seeds were recovered in situ and treated similarly to calcined bones when possible, but the majority of samples were collected during flotation. These samples were initially placed in pill-casings for several weeks, but were promptly wrapped in aluminum foil and placed in glass vials when the samples arrived at the ${ }^{14} \mathrm{C}$ laboratory. Each layer (labeled $\mathrm{A} 1-\mathrm{A} 10$ from top to bottom, see Figure 1) included 3 calcined bone samples, and up to 2 samples each of charcoal and seeds. Variation in the number of charcoal and seed samples reflects the presence or absence of these materials in the sediments. Calcined bone samples were long bone shaft fragments from medium to large-sized ungulates. Based on the representation of remains at the site, they were most likely fragments of sheep or goat femurs (Starkovich, forthcoming). Archaeological units observed in-field called baskets (Z123, Z132, and Z139) are included for clarity because multiple ${ }^{14} \mathrm{C}$ samples fit within each archaeological layer. In addition, 4 calcined bones were selected from each of the 4 layers through the sequence (A3, A5, A7, and A9) to test whether or not surface contamination is an issue with the samples. These 4 samples were each divided into 3 parts so they could be subjected to the experimental protocol outlined below, as well as the "standard" pretreatment method applied to other calcined bones in the sequence, and FTIR measurements.

\section{Pretreatment of Organic Remains}

The pretreatment of charcoal and seed samples followed standard NSF-Arizona AMS Lab ABA protocols (Lange et al. 2001). Samples were first soaked in $1 \mathrm{M} \mathrm{HCl}$ for $24 \mathrm{hr}$ at $80{ }^{\circ} \mathrm{C}$, then soaked in $1 \mathrm{M} \mathrm{NaOH}$ for 1 or more days at $80^{\circ} \mathrm{C}$, and finally soaked in $1 \mathrm{M} \mathrm{HCl}$, with each step punctuated by rinsing with deionized water. Samples were combusted under vacuum in the presence of $\mathrm{CuO}$

followed by cryogenic separation of $\mathrm{CO}_{2}$ from the combustion gasses. $\delta^{13} \mathrm{C}$ values were measured 


\section{B M Starkovich et al.}

Figure 1 Schematic representation of the sediment column from which paired ${ }^{14} \mathrm{C}$ samples were collected. Note that sample collection began about $50 \mathrm{~cm}$ below the surface, in fully intact sediments. Numbers to the right designated with the letter "Z" represent archaeological baskets. Depth is expressed in meters above sea level ( $\mathrm{m}$ asl).

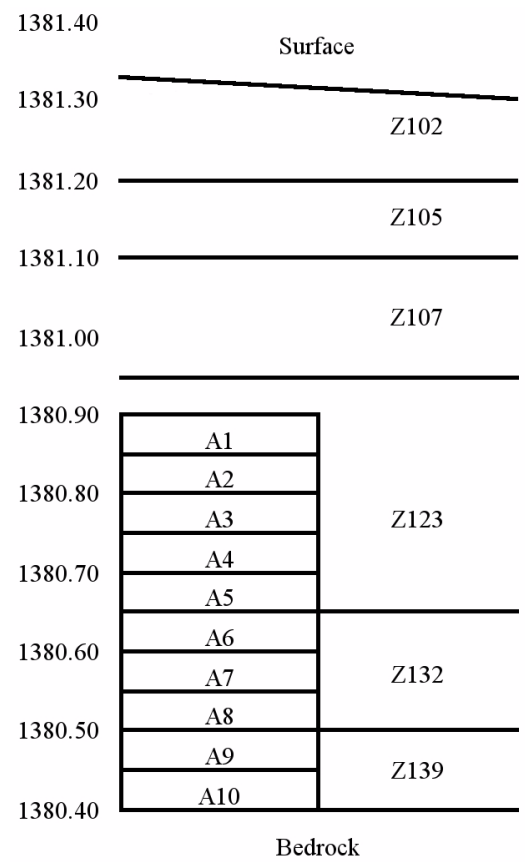

off-line using a dual-inlet stable isotope mass spectrometer, and the remaining $\mathrm{CO}_{2}$ was converted to graphite for accelerator mass spectrometry (AMS) measurement by the method of Slota et al. (1987). Results are background-subtracted and isotope fractionation-corrected. The results are reported in ${ }^{14} \mathrm{C}$ yr BP and the calibrated age ranges expressed in years BCE at the $95.4 \%$ confidence interval (Table 1). Results are calibrated using the IntCal09 curve (Reimer et al. 2009) in OxCal v 4.1 (Bronk Ramsey 2009).

\section{Calcined Bone Experiments and Pretreatment}

In selecting calcined bones for dating, visual inspections were conducted to determine the quality of the samples. Only bones with no visible recent cracks or breaks that were entirely white with no traces of gray were chosen. Van Strydonck et al. (2009) determined that in certain environmental conditions, carbonate exchange can occur between the surface of calcined bone and the surrounding sediments. In order to test for this possibility, a series of time-based hydrolysis measurements were taken on 4 samples (A3, A5, A7, and A9) throughout the stratigraphic sequence. Each of the 4 samples were sonicated in deionized water, then ground to 0.25 to $1.0 \mathrm{~mm}$ using an agate mortar and pestle. The unpretreated samples were placed in a closed vessel where they were reacted with phosphoric acid to release $\mathrm{CO}_{2}$. Carbon dioxide measurements were collected at specific time intervals throughout the reaction (Table 2). The purpose of the time series hydrolysis was to determine if there was directional change in either the ${ }^{14} \mathrm{C}$ dates or $\delta^{13} \mathrm{C}$ values "stratigraphically" through a single bone. That is, the first measurement mostly reflects surface conditions, and each subsequent measurement is from deeper within the bone. Since the bones were ground, the measurements do not reflect pure "stratigraphic" measurements on the samples as seen in Van Strydonck et al. (2009), but they are stratigraphic in the sense that the first measurements are from the outside of ground fragments, and later measurements are from deeper within the powders. In this study, grinding was necessary due to prohibitively long reaction times for whole pieces and concerns over leaving samples on the line for days at a time. 


\section{${ }^{14}$ C Dates from the Sanctuary of Zeus on Mt. Lykaion}

Table 1 Paired ${ }^{14} \mathrm{C}$ dates and $\delta^{13} \mathrm{C}$ values from the Sanctuary of Zeus on Mt. Lykaion. *Large samples were

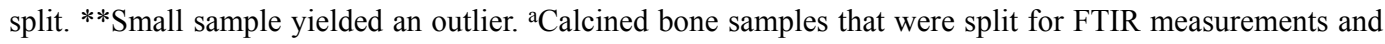
time series hydrolysis.

\begin{tabular}{|c|c|c|c|c|c|c|c|c|c|}
\hline Lab \# & $\begin{array}{l}\text { Sample } \\
\text { ID }\end{array}$ & Material & $\begin{array}{l}\text { Treated } \\
\text { sample } \\
(\mathrm{mg})\end{array}$ & $\begin{array}{l}\mathrm{CO}_{2} \\
\text { yield } \\
(\mathrm{cc}) \\
\end{array}$ & $\begin{array}{l}\text { Mass } \\
\text { of C } \\
(\mathrm{mg})\end{array}$ & $\begin{array}{l}\% \\
\text { yield }\end{array}$ & $\begin{array}{l}\delta^{13} \mathrm{C} \\
(\%)\end{array}$ & $\begin{array}{l}{ }^{14} \mathrm{C} \text { age } \\
\text { BP }\end{array}$ & $\begin{array}{l}\text { cal age BCE } \\
\text { (95.4\% conf.) }\end{array}$ \\
\hline AA97258 & A1a-1 & Charcoal & 1.68 & 1.97 & 0.96 & 57.40 & -25.9 & $2435 \pm 42$ & $753-404$ \\
\hline AA97259 & A1a-2 & Charcoal & 1.50 & 0.90 & 0.90 & 60.00 & -26.6 & $2512 \pm 39$ & $796-426$ \\
\hline AA97260 & A1b-1 & Seeds & 2.25 & 2.68 & 1.31 & 58.30 & -19.2 & $2512 \pm 39$ & $796-426$ \\
\hline AA97261 & Alb-2 & Seeds & 1.93 & 2.42 & 1.19 & 61.40 & -24.2 & $2509 \pm 38$ & $794-426$ \\
\hline AA97262 & Alc-1 & Calcined bone & 869.30 & 8.88 & 2.78 & 0.50 & -23.0 & $2517 \pm 48$ & $799-418$ \\
\hline AA97263 & A1c-2 & Calcined bone & 232.54 & 1.65 & 0.81 & 0.35 & -27.1 & $2503 \pm 48$ & $793-417$ \\
\hline AA97264 & A1c-3 & Calcined bone & 335.54 & 2.11 & 1.03 & 0.31 & -24.1 & $2515 \pm 48$ & $798-418$ \\
\hline AA97266 & A2a-1 & Charcoal & 1.52 & 1.69 & 0.83 & 54.60 & -23.2 & $2472 \pm 39$ & $766-414$ \\
\hline AA97267 & A2a-2 & Charcoal & 1.46 & 2.12 & 1.04 & 71.20 & -26.1 & $2454 \pm 39$ & $756-410$ \\
\hline AA97268 & A2c-1 & Calcined bone & 476.20 & 2.40 & 1.17 & 0.25 & -25.2 & $2510 \pm 48$ & $796-417$ \\
\hline AA97269 & $\mathrm{A} 2 \mathrm{c}-2$ & Calcined bone & 433.07 & 2.75 & 1.35 & 0.31 & -23.9 & $2564 \pm 48$ & $818-538$ \\
\hline AA97270 & $\mathrm{A} 2 \mathrm{c}-3$ & Calcined bone & 296.90 & 2.03 & 1.00 & 0.34 & -26.6 & $2497 \pm 48$ & $789-417$ \\
\hline AA97272 & A3a-1 & Charcoal & 2.24 & 2.64 & 1.29 & 57.70 & -24.5 & $2558 \pm 39$ & $808-511$ \\
\hline AA97273 & A3a-2 & Charcoal & 1.75 & 2.21 & 1.08 & 61.80 & -25.4 & $2501 \pm 44$ & $791-417$ \\
\hline AA97274 & A3b-1 & Seeds & 1.99 & 2.46 & 1.21 & 61.00 & -24.8 & $2574 \pm 39$ & $815-548$ \\
\hline AA97275 & A $3 b-2$ & Seeds & 2.35 & 3.18 & 1.56 & 66.20 & -21.0 & $2499 \pm 38$ & $790-418$ \\
\hline AA97276 & A3c-1 & Calcined bone & 758.79 & 2.55 & 1.25 & 0.16 & -20.8 & $2654 \pm 49$ & $916-768$ \\
\hline AA97277 & $\mathrm{A} 3 \mathrm{c}-2$ & Calcined bone & 837.53 & $4.89^{*}$ & 1.42 & 0.29 & -24.4 & $2757 \pm 49$ & $1011-811$ \\
\hline AA97278 & $\mathrm{A} 3 \mathrm{c}-3$ & Calcined bone $\mathrm{a}^{\mathrm{a}}$ & 272.82 & 2.25 & 1.10 & 0.40 & -23.4 & $2525 \pm 56$ & $802-417$ \\
\hline AA97280 & A4a-1 & Charcoal & 1.72 & 2.26 & 1.11 & 64.00 & -24.2 & $2541 \pm 38$ & $802-540$ \\
\hline AA97281 & A4b-1 & Seeds & 2.07 & 2.73 & 1.34 & 65.00 & -23.4 & $2442 \pm 37$ & $753-407$ \\
\hline AA97282 & A4b-2 & Seeds & 2.16 & 3.07 & 1.51 & 70.00 & -21.9 & $2522 \pm 37$ & $796-522$ \\
\hline AA97283 & $\mathrm{A} 4 \mathrm{c}-1$ & Calcined bone & 840.58 & $4.67 *$ & 1.35 & 0.27 & -21.3 & $2582 \pm 49$ & $836-540$ \\
\hline AA97284 & $\mathrm{A} 4 \mathrm{c}-2$ & Calcined bone & 811.06 & $5.42 *$ & 1.57 & 0.32 & -20.4 & $2457 \pm 48$ & $761-410$ \\
\hline AA97285 & $\mathrm{A} 4 \mathrm{c}-3$ & Calcined bone & 110.84 & 0.38 & 0.19 & 0.16 & -25.0 & $2696 \pm 93$ & $1121-551$ \\
\hline AA97287 & A5a-1 & Charcoal & 1.76 & 2.41 & 1.18 & 67.00 & -24.7 & $2680 \pm 38$ & $906-796$ \\
\hline AA97288 & A5a-2 & Charcoal & 1.74 & 2.41 & 1.18 & 68.00 & -25.1 & $2481 \pm 46$ & $772-415$ \\
\hline AA97289 & A5b-1 & Seeds & 1.76 & 2.81 & 1.38 & 78.00 & -22.9 & $2495 \pm 37$ & $787-418$ \\
\hline AA97290 & A $5 b-2$ & Seeds & 1.93 & 2.80 & 1.37 & 71.00 & -24.1 & $2520 \pm 38$ & $795-521$ \\
\hline AA97291 & A5c-1 & Calcined bone & 702.03 & $4.86^{*}$ & 1.40 & 0.34 & -23.3 & $2489 \pm 48$ & $781-416$ \\
\hline AA97292 & $\mathrm{A} 5 \mathrm{c}-2$ & Calcined bone & 490.91 & $5.14 *$ & 1.49 & 0.50 & -23.0 & $2532 \pm 48$ & $805-426$ \\
\hline AA97293 & A $5 c-3$ & Calcined bone $^{\mathrm{a}}$ & 55.68 & 0.25 & 0.12 & 0.22 & -24.6 & $2860 \pm 130$ & $1386-807^{* *}$ \\
\hline AA97295 & A6a-1 & Charcoal & 1.76 & 2.59 & 1.27 & 72.00 & -25.9 & $2749 \pm 53$ & $1011-806$ \\
\hline AA97296 & A6b-1 & Seeds & 1.90 & 2.74 & 1.34 & 71.00 & -24.0 & $2549 \pm 37$ & $804-543$ \\
\hline AA97297 & A6b-2 & Seeds & 1.90 & 2.54 & 1.24 & 65.00 & -22.9 & $2604 \pm 37$ & $839-594$ \\
\hline AA97298 & A6c-1 & Calcined bone & 1230.53 & 1.37 & 0.67 & 0.05 & -24.9 & $2553 \pm 49$ & $811-521$ \\
\hline AA97299 & A $6 c-2$ & Calcined bone & 531.36 & $3.95^{*}$ & 1.14 & 0.36 & -23.5 & $2607 \pm 49$ & $896-551$ \\
\hline AA97300 & A $6 \mathrm{c}-3$ & Calcined bone & 442.68 & 1.86 & 0.91 & 0.21 & -26.6 & $2649 \pm 48$ & $912-767$ \\
\hline AA97302 & A7a-1 & Charcoal & 1.64 & 2.51 & 1.23 & 75.00 & -25.4 & $2640 \pm 37$ & $896-773$ \\
\hline AA97303 & A7b-1 & Seeds & 1.85 & 2.55 & 1.25 & 67.00 & -24.9 & $2544 \pm 37$ & $803-541$ \\
\hline AA97304 & A $7 b-2$ & Seeds & 1.93 & 2.54 & 1.24 & 64.00 & -24.7 & $2553 \pm 38$ & $806-543$ \\
\hline AA97305 & A7c-1 & Calcined bone & 439.50 & 2.64 & 1.29 & 0.29 & -20.8 & $2596 \pm 48$ & $891-544$ \\
\hline AA97306 & $\mathrm{A} 7 \mathrm{c}-2$ & Calcined bone & 545.77 & 3.40 & 1.67 & 0.31 & -26.4 & $2793 \pm 49$ & $1111-826$ \\
\hline AA97307 & A $7 c-3$ & Calcined bone ${ }^{a}$ & 231.39 & 1.14 & 0.56 & 0.29 & -22.6 & $2590 \pm 59$ & $896-524$ \\
\hline AA97309 & A8a-1 & Charcoal & 0.40 & 0.34 & 0.17 & 41.00 & -21.7 & $2150 \pm 74$ & $387-4^{* *}$ \\
\hline AA97310 & A $8 b-1$ & Seeds & 1.98 & 2.76 & 1.35 & 68.00 & -22.4 & $2517 \pm 37$ & $794-521$ \\
\hline AA97311 & A $8 b-2$ & Seeds & 1.84 & 2.34 & 1.14 & 62.00 & -24.1 & $2507 \pm 37$ & $793-427$ \\
\hline AA97312 & A $8 \mathrm{c}-1$ & Calcined bone & 390.56 & 1.11 & 0.55 & 0.14 & -23.3 & $2626 \pm 49$ & $907-593$ \\
\hline AA97313 & $\mathrm{A} 8 \mathrm{c}-2$ & Calcined bone & 200.80 & 0.91 & 0.44 & 0.22 & -20.7 & $2789 \pm 54$ & $1111-819$ \\
\hline AA97314 & A8c-3 & Calcined bone & 260.05 & 0.70 & 0.34 & 0.13 & -24.4 & $2625 \pm 64$ & $925-543$ \\
\hline
\end{tabular}




\section{B M Starkovich et al.}

Table 1 Paired ${ }^{14} \mathrm{C}$ dates and $\delta^{13} \mathrm{C}$ values from the Sanctuary of Zeus on Mt. Lykaion. *Large samples were

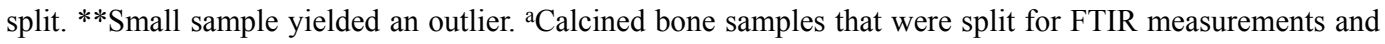
time series hydrolysis. (Continued)

\begin{tabular}{|c|c|c|c|c|c|c|c|c|c|}
\hline Lab \# & $\begin{array}{l}\text { Sample } \\
\text { ID }\end{array}$ & Material & $\begin{array}{l}\text { Treated } \\
\text { sample } \\
(\mathrm{mg})\end{array}$ & $\begin{array}{l}\mathrm{CO}_{2} \\
\text { yield } \\
\text { (cc) }\end{array}$ & $\begin{array}{l}\text { Mass } \\
\text { of C } \\
(\mathrm{mg}) \\
\end{array}$ & $\begin{array}{l}\% \\
\text { yield }\end{array}$ & $\begin{array}{l}\delta^{13} \mathrm{C} \\
(\%)\end{array}$ & $\begin{array}{l}{ }^{14} \mathrm{C} \text { age } \\
\mathrm{BP}\end{array}$ & $\begin{array}{l}\text { cal age BCE } \\
(95.4 \% \text { conf.) }\end{array}$ \\
\hline AA97316 & A9a-1 & Charcoal & 1.59 & 1.98 & 0.97 & 61.00 & -25.3 & $2670 \pm 38$ & $901-795$ \\
\hline AA97317 & A9c-1 & Calcined bone ${ }^{a}$ & 111.12 & 0.44 & 0.21 & 0.20 & -25.0 & $2781 \pm 99$ & $1257-793$ \\
\hline AA97318 & A9c-2 & Calcined bone & 538.45 & $4.49 *$ & 1.28 & 0.40 & -25.6 & $3189 \pm 51$ & $1608-1324$ \\
\hline AA97319 & A9c-3 & Calcined bone & 463.69 & 3.64 & 1.78 & 0.38 & -23.3 & $2715 \pm 49$ & $976-798$ \\
\hline AA97321 & A10a-1 & Charcoal & 1.50 & 1.98 & 0.97 & 65.00 & -24.5 & $3061 \pm 38$ & $1421-1215$ \\
\hline AA97322 & A10a-2 & Charcoal & 1.10 & 1.37 & 0.67 & 0.67 & -23.5 & $2777 \pm 38$ & $1012-832$ \\
\hline AA97323 & A10b-1 & Seeds & 1.76 & 1.20 & 1.20 & 68.00 & -22.2 & $2530 \pm 37$ & $798-538$ \\
\hline AA97324 & A10c-1 & Calcined bone & 206.72 & 1.01 & 0.49 & 0.24 & -24.5 & $2678 \pm 49$ & $928-787$ \\
\hline AA97325 & A10c-2 & Calcined bone & 201.80 & 1.23 & 0.60 & 0.30 & -18.6 & $2758 \pm 49$ & $1011-811$ \\
\hline AA97326 & A $10 c-3$ & Calcined bone & 77.00 & 0.52 & 0.25 & 0.33 & -23.4 & $3237 \pm 86$ & $1739-1316$ \\
\hline
\end{tabular}

Calcined bone dating of individual samples followed recommendations outlined in Van Strydonck et al. (2009). Samples were visually inspected and selected based on the severity of burning and lack of recent damage. They were then sonicated in deionized water to remove surface dirt. Though there was no reason to believe that exchange had occurred between bone surfaces and the surrounding sedimentary matrix (see below), each sample was etched with $1 \% \mathrm{HCl}$ for $15 \mathrm{~min}$ to $2 \mathrm{hr}$ at $80^{\circ} \mathrm{C}$. Differences in etching time reflect sample size and concerns that small samples would be destroyed with prolonged etching. Samples were then ground to 0.25 to $1.0 \mathrm{~mm}$ and soaked in $1 \mathrm{M}$ acetic acid at room temperature overnight. After rinsing with deionized water, samples were reacted with phosphoric acid and $\mathrm{CO}_{2}$ was collected for graphitization and AMS measurement. Results are again reported in yr BP and calibrated age ranges BCE, with a 95.4\% confidence interval (Table 1).

Table 2 Time series hydrolysis measurements from samples A3, A5, A7, and A9. Note that time measurements were not the same for all samples, indicating an improvement in the methodology as the experiments continued. *Initial sample yielded $3.69 \mathrm{mg} \mathrm{C}$; smaller sample reflects split.

\begin{tabular}{|c|c|c|c|c|c|c|c|c|c|c|}
\hline $\begin{array}{l}\text { Initial } \\
\text { sample } \\
(\mathrm{mg})\end{array}$ & Lab \# & $\begin{array}{l}\mathrm{CO}_{2} \\
\text { yield } \\
\text { (cc) }\end{array}$ & $\begin{array}{l}\% \mathrm{CO}_{2} \\
\text { yield }\end{array}$ & $\begin{array}{l}\% \mathrm{CO}_{2} \\
\text { additive }\end{array}$ & $\begin{array}{l}\text { Mass } \\
\text { of C } \\
(\mathrm{mg})\end{array}$ & $\begin{array}{l}\% \\
\text { yield }\end{array}$ & $\begin{array}{l}\delta^{13} \mathrm{C} \\
(\%)\end{array}$ & $\begin{array}{l}{ }^{14} \mathrm{C} \text { age } \\
\text { BP }\end{array}$ & $\begin{array}{l}\text { Cal age } \\
\text { BCE }\end{array}$ & $\begin{array}{l}\text { Time } \\
\text { series } \\
(\mathrm{min})\end{array}$ \\
\hline \multicolumn{11}{|c|}{ Sample A3 } \\
\hline 1425.70 & AA97265 & 7.53 & 57.75 & 57.75 & $2.18 *$ & 0.26 & -22.0 & $2537 \pm 49$ & $659 \pm 91$ & 20 \\
\hline 1425.70 & AA97271 & 3.07 & 23.54 & 81.29 & 1.50 & 0.11 & -22.1 & $2552 \pm 49$ & $675 \pm 91$ & 40 \\
\hline 1425.70 & AA97279 & 1.89 & 14.49 & 95.78 & 0.93 & 0.07 & -22.3 & 2505 & $632 \pm 94$ & 120 \\
\hline 1425.70 & AA97286 & 0.55 & 4.22 & 100.00 & 0.27 & 0.02 & -22.2 & 2579 & $688 \pm 113$ & 240 \\
\hline Total & & 13.04 & & & 4.21 & & & & & \\
\hline \multicolumn{11}{|c|}{ Sample A5 } \\
\hline 465.30 & AA97294 & 0.55 & 27.64 & 27.64 & 0.27 & 0.06 & -17.8 & 232 & 428 & 10 \\
\hline 465.30 & AA97301 & 0.65 & 32.66 & 60.30 & 0.32 & 0.07 & -19.1 & 225 & & 25 \\
\hline 30 & AA97308 & 0.53 & 26.63 & 86.93 & 0.26 & 0.06 & -18.4 & 220 & 260 & 90 \\
\hline 465.30 & AA97315 & 0.26 & 13.07 & 100.00 & 0.13 & 0.03 & -19.0 & $2460 \pm 110$ & $587 \pm 134$ & 240 \\
\hline Total & & 1.99 & & & 0.98 & & & & & \\
\hline \multicolumn{11}{|c|}{ Sample A7 } \\
\hline 1673.90 & AA97320 & 1.67 & 16.15 & 16.15 & 0.75 & 0.04 & -18.7 & $2460 \pm 48$ & $592 \pm 104$ & 5 \\
\hline 1673.90 & AA97327 & 3.38 & 32.69 & 48.84 & 1.67 & 0.10 & -21.0 & $2497=$ & $625 \pm 96$ & 20 \\
\hline 1673.90 & AA98405 & 3.62 & 35.01 & 83.85 & 1.78 & 0.11 & -21.1 & $2457 \pm 48$ & $589 \pm 105$ & 90 \\
\hline 1673.90 & AA98406 & 1.67 & 16.15 & 100.00 & 0.82 & 0.05 & -21.0 & $2457 \pm 48$ & $589 \pm 105$ & 240 \\
\hline Total & & 10.34 & & & 5.02 & & & & & \\
\hline
\end{tabular}


${ }^{14}$ C Dates from the Sanctuary of Zeus on Mt. Lykaion

Table 2 Time series hydrolysis measurements from samples A3, A5, A7, and A9. Note that time measurements were not the same for all samples, indicating an improvement in the methodology as the experiments continued. *Initial sample yielded $3.69 \mathrm{mg} \mathrm{C}$; smaller sample reflects split. (Continued)

\begin{tabular}{|c|c|c|c|c|c|c|c|c|c|c|}
\hline $\begin{array}{l}\text { Initial } \\
\text { sample } \\
\text { (mg) }\end{array}$ & Lab \# & $\begin{array}{l}\mathrm{CO}_{2} \\
\text { yield } \\
\text { (cc) }\end{array}$ & $\begin{array}{l}\% \mathrm{CO}_{2} \\
\text { yield }\end{array}$ & $\begin{array}{l}\% \mathrm{CO}_{2} \\
\text { additive }\end{array}$ & $\begin{array}{l}\text { Mass } \\
\text { of C } \\
(\mathrm{mg})\end{array}$ & $\begin{array}{l}\% \\
\text { yield }\end{array}$ & $\begin{array}{l}\delta^{13} \mathrm{C} \\
(\%)\end{array}$ & $\begin{array}{l}{ }^{14} \mathrm{C} \text { age } \\
\mathrm{BP}\end{array}$ & $\begin{array}{l}\text { Cal age } \\
\text { BCE }\end{array}$ & $\begin{array}{l}\text { Time } \\
\text { series } \\
(\mathrm{min})\end{array}$ \\
\hline \multicolumn{11}{|c|}{ Sample A9 } \\
\hline 1020.40 & AA98407 & 1.22 & 23.51 & 23.51 & 0.60 & 0.06 & -20.8 & $2352 \pm 48$ & 463 & 5 \\
\hline 1020.40 & AA98408 & 1.51 & 29.09 & 52.60 & 0.74 & 0.07 & -23.1 & $2539 \pm 49$ & 661 & 20 \\
\hline 1020.40 & AA98409 & 1.84 & 35.45 & 88.05 & 0.90 & 0.09 & -23.1 & $2522 \pm 48$ & 646 & 90 \\
\hline 1020.40 & AA98410 & 0.62 & 11.95 & 100.00 & 0.30 & 0.03 & -23.3 & $2580 \pm 69$ & 691 & 240 \\
\hline Total & & 5.19 & & & 2.54 & & & & & \\
\hline
\end{tabular}

\section{Fourier Transform Infrared (FTIR) Measurements}

Portions of the 4 bone samples used for the time series hydrolysis (A3, A5, A7, and A9) were prepared for FTIR analysis using the method of Wright and Schwarcz (1996). Twenty-five to $40 \mathrm{mg}$ of bone were crushed to less than $1 \mathrm{~mm}$ and soaked in $1.5 \mathrm{~mL}$ of $1.5 \%$ sodium hypochlorite for $48 \mathrm{hr}$ at room temperature. The powders were washed 3 times in deionized water by centrifugation, and then placed in $1 \mathrm{M}$ acetic acid for $24 \mathrm{hr}$ at room temperature. Acetic acid was removed by another 3 washes with deionized water, and the powders dried at $70{ }^{\circ} \mathrm{C}$ for $24 \mathrm{hr}$. Samples were gently crushed to $<0.1-\mathrm{mm}$ particles using an agate mortar and pestle before loading into the spectrometer.

FTIR measurements were conducted using a Nicolet Avatar 360 spectrometer in attenuated reflectance mode (ATR). The ATR diamond cell was set for a 1-mm path length. Scans spanned 4000 to $525 \mathrm{~cm}^{-1}$ at $6 \mathrm{~cm}^{-1}$ resolution, and each sample was scanned 64 times. Background scans were collected from air. The scans shown in Figure 2 are baseline- and ATR-corrected.

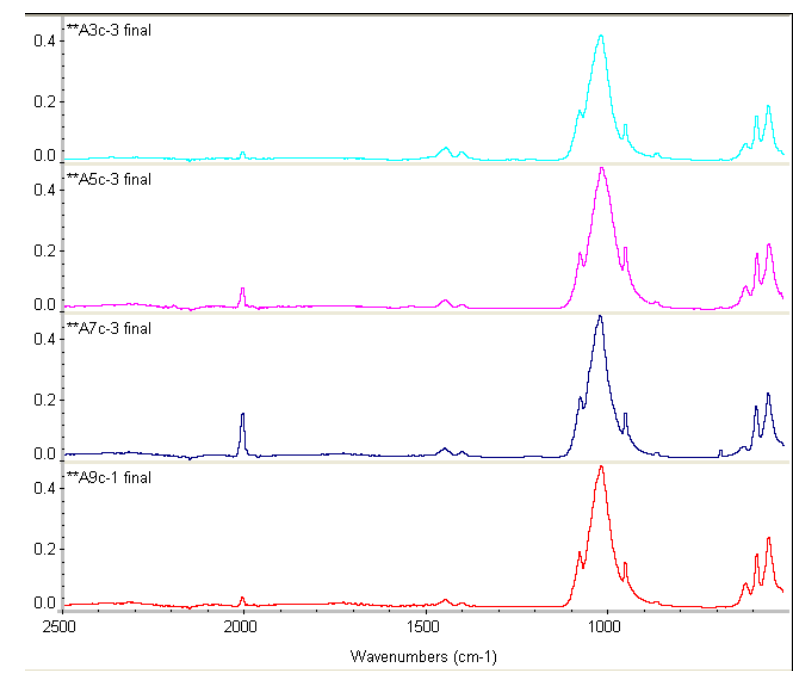

Figure 2 FTIR spectra of 4 bone powders from the altar site of Mt. Lykaion. Splitting factors (SF) of 5.3, 4.8, 5.6, and 5.7 were measured for samples A3, A5, A7, and A9, respectively. These SF values indicate significant calcination. The small peaks at 1415 and $874 \mathrm{~cm}^{-1}$ indicate a loss of carbonate, consistent with carbonate yields on hydrolysis. The small peaks at $2012 \mathrm{~cm}^{-1}$ are cyanamide-apatite peaks; Hüls et al. (2010) suggested this indicates cremation occurred with flesh on the bones. 


\section{B M Starkovich et al.}

Infrared splitting factor (SF) calculations were according to Weiner and Bar-Yosef (1990), Olsen et al. (2008), and Hüls et al. (2010), that is, $\mathrm{SF}=(\mathrm{A} 603+\mathrm{A} 565) /(\mathrm{A}$ valley). Peak and trough heights for our SF calculations were made on baseline and ATR-corrected spectra using a linear baseline drawn between 750 and $525 \mathrm{~cm}^{-1}$, not 750 to $495 \mathrm{~cm}^{-1}$ used by the cited authors. The alternative baseline was required because high noise levels in our spectra $<520 \mathrm{~cm}^{-1}$ obscured the $495 \mathrm{~cm}^{-1}$ absorbance. We estimate the slightly higher baseline had the effect of increasing our SF calculations by 0.2 . Quoted values are corrected by this amount. In our spectra, peaks were measured at A 600 $602 \mathrm{~cm}^{-1}$ and A $567-569 \mathrm{~cm}^{-1}$. Valley minima were measured at $590 \mathrm{~cm}^{-1}$.

\section{RESULTS AND DISCUSSION}

The results from the time series hydrolysis experiments are presented in Table 2 and Figure 3. Van Strydonck et al. $(2005,2009)$ found clear evidence of surface contamination by noticeably older ${ }^{14} \mathrm{C}$ dates and more negative $\delta^{13} \mathrm{C}$ values on the outer layers of bones that experienced some surface contamination. The methods used here differ slightly from the above-mentioned studies, in that the Mt. Lykaion samples were ground; however, time series measurements are still expected to produce directional trends if surface contamination was a problem. There are no major trends for ${ }^{14} \mathrm{C}$ values through the "stratigraphic" sequence of any of the 4 Mt. Lykaion bones. There appears to be a mild directional trend for sample A9 that is mostly driven by a younger date on the outermost measurement of the bone, which may indicate a small amount of exchange between the bone surface and surrounding matrix. No trends are apparent for $\delta^{13} \mathrm{C}$ values for samples $\mathrm{A} 3$ or $\mathrm{A} 5$, though samples A7 and A9 display a substantial drop between the first and second measurements (Table 2, Figure 3).

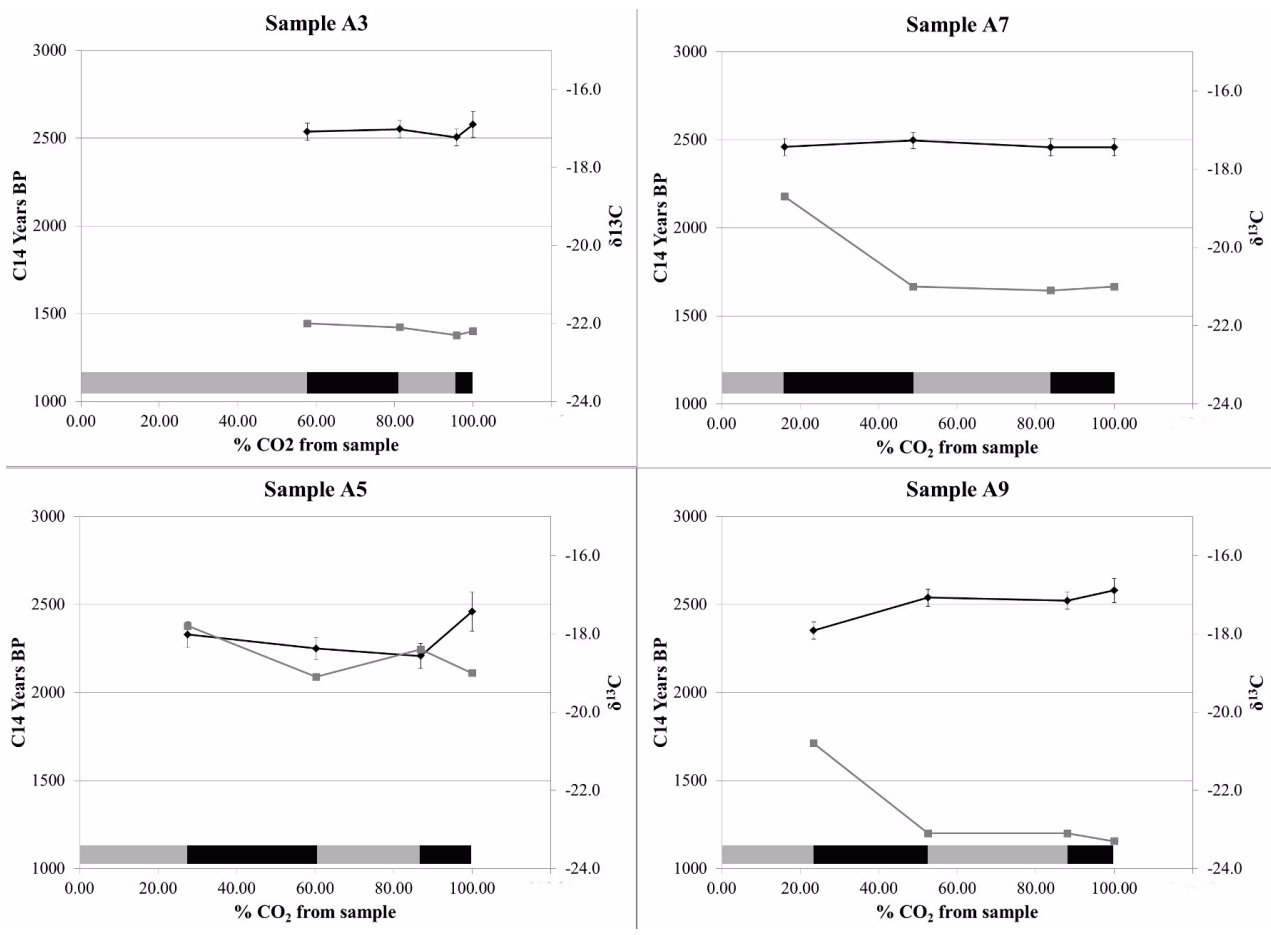

Figure 3 Results of time series hydrolysis for samples A3, A5, A7, and A9. Black lines are ${ }^{14} \mathrm{C}$ dates and gray lines are $\delta^{13} \mathrm{C}$ values. Gray and black bars at the bottom of each graph indicate different fractions of measured $\mathrm{CO}_{2}$. None of the samples show a major trend in ${ }^{14} \mathrm{C}$ dates through different layers of bone. Data from Table 2. 
Paired ${ }^{14} \mathrm{C}$ dates by layer are reported in Table 1 and Figure 4 . In general, there is very good agreement between calcined bone dates and ${ }^{14} \mathrm{C}$ values for seeds and charcoal. There are several noteworthy observations. First, dates on seeds are very consistent with one another throughout the sequence. This is not the case with charcoal and calcined bone. Rather, there is increasing variation in charcoal and bone dates in the lower sample sets, corresponding with archaeological basket Z139. It is possible that the lack of a similar trend in seed dates is due to the fact that only 1 seed was available for dating from basket Z139, and more varied dates would be apparent if the sample were larger. Another possibility is that small seeds from the upper archaeological units moved through the sedimentary sequence, at least in the case of sample A10b-1 (Table 1).

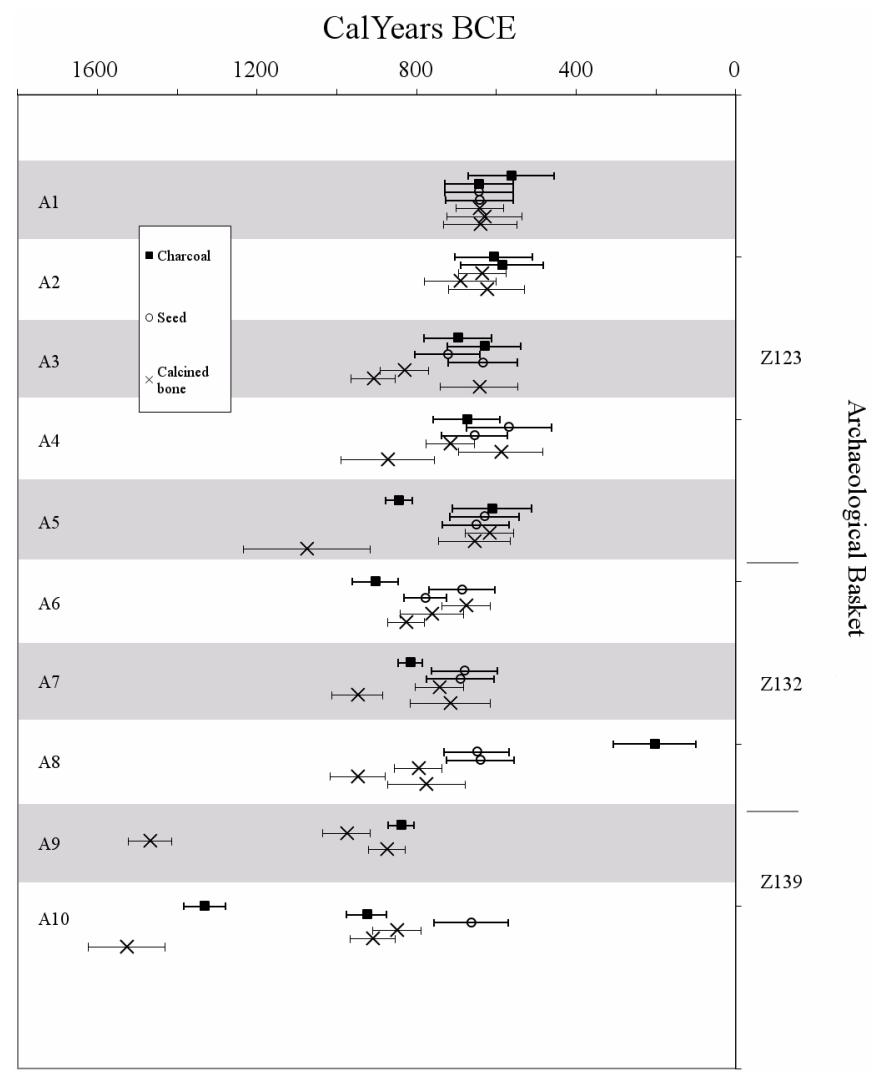

Figure 4 Mean calibrated dates and error ranges for each of the 60 paired samples from the altar. Alternating gray and white bars indicate different samples from the sediment column. Note that within a sample number (e.g. A1), samples are not in stratigraphic order because they all came from the same $25 \times$ $25 \times 5 \mathrm{~cm}$ square. Archaeological baskets are indicated to the right. A charcoal date from sample A8 yielded a very young outlier. Variation increases deeper in the sequence, but several bone and charcoal dates indicate that the altar was in use by the Mycenaean period.

The next observation is that there is a hiatus in dates between about 1000 and $1300 \mathrm{cal} \mathrm{BCE}$. This could represent a gap in the use of the site, but more likely reflects the small sample universe from which ${ }^{14} \mathrm{C}$ dates were collected. The stratigraphic column is only $25 \times 25 \mathrm{~cm}$, from a mountaintop that is approximately $30 \mathrm{~m}$ in diameter and covered in cultural remains (Romano and Voyatzis, 


\section{B M Starkovich et al.}

forthcoming). It is possible that different areas of the altar were used in different time periods, or that cleaning activities occurred during certain phases. Analyses of ceramics from the column and surrounding contexts are currently underway to determine if this hiatus is also apparent in the cultural remains, in order to test the hypothesis that this gap in dates represents changes in the use of different parts of the site. Preliminary results of the ceramic analysis indicate that activity continued during most, if not all, of the period between 1000-1300 BCE (Romano and Voyatzis, forthcoming).

The range of dates from the altar must also be addressed. No dates later than $550 \mathrm{cal} \mathrm{BCE}$ were recorded, with the exception of the outlier from sample A8. This contrasts with the ceramic and other artifactual evidence, which indicates that the altar was used until the 4th century BCE. Recall, however, that due to mixing of the top layers of the site, samples were intentionally collected starting about $50 \mathrm{~cm}$ below the surface, in an attempt to establish the success of the method in this context. The 3 earliest dates from the site are noteworthy, both for their antiquity in this context and because they corroborate one another quite well. They place the earliest use of the altar firmly within the Mycenaean period (1600-1100 BCE).

Throughout the sequence, $\delta^{13} \mathrm{C}$ values on calcined bones were extremely low $(-18.6$ to -27.1 ; Table 1). This is consistent with calcined bone $\delta^{13} \mathrm{C}$ values recorded by other authors (Van Strydonck et al. 2005, 2009; Olsen et al. 2008; Zazzo et al. 2009). It is clear from the faunal analysis that the bones in this study all represent terrestrial herbivores, mostly sheep and goats (Starkovich, forthcoming), and this is reflected in the low $\delta^{13} \mathrm{C}$ values. Early studies hypothesized that $\delta^{13} \mathrm{C}$ values from calcined bone could be used to reconstruct past diets (Olsen et al. 2008), but it was later shown that carbon exchange with the environment during combustion, as well as possible fractionation during the cremation process, makes such reconstructions unreliable (Zazzo et al. 2009; Hüls et al. 2010; Van Strydonck et al. 2010).

The 4 FTIR Spectra are shown in Figure 2. The reduced absorbance at 874 and $1415 \mathrm{~cm}^{-1}$ in all 4 samples indicates a loss of $\mathrm{CO}_{3}$ compared to levels found in modern bone. Reduction in the structural carbonate content is characteristic of calcined bone (Stiner et al. 1995). This observation is consistent with the low carbon yields found after hydrolysis of the bone mineral for ${ }^{14} \mathrm{C}$ measurement (Table 1).

Splitting factors for samples A3, A5, A7, and A9 were calculated as 5.3, 4.8, 5.6, 5.7, respectively. All values indicate significant increase in the orderliness of apatite crystals compared with unheated/1M acetic acid-treated bone (see Wright and Schwarz 1996). High splitting factor values are consistent with calcined bone values found by other authors (e.g. Olsen et al. 2008; Thompson et al. 2009; Lebon et al. 2010; Zazzo et al. 2009). The loss of structural carbonate, combined with the SF measurements corroborate the visual assessment that the bones are indeed calcined and therefore likely function as closed systems that resist groundwater carbon exchange.

All 4 samples also show absorption peaks at $2012 \mathrm{~cm}^{-1}$ indicating the presence of cyanamide-apatite bonds. Hüls et al. (2010) and Van Strydonck et al. (2010) showed experimentally that these peaks appear when bone and flesh are combusted together. Habelitz et al. $(1999,2001)$ documented nitrogen and carbon incorporation into low carbon synthetic apatite crystals as cyanamide groups, if the apatites were heated to 800 to $1300^{\circ} \mathrm{C}$ in an ammonia atmosphere, in the presence of graphite. If the $2012 \mathrm{~cm}^{-1}$ peak is a marker for cremation with attached or associated body tissues (as Hüls et al. 2010 suggests), its presence in the FTIR spectra of 4 out of 4 of Mt. Lykaion animal bones, each from different stratigraphic locations, suggests the large quantities of caprine legs and tails were burnt with flesh on them, over a significant period of time. This archaeological evidence is striking as it seems to reflect practices described in the Homeric epics. 
${ }^{14}$ C Dates from the Sanctuary of Zeus on Mt. Lykaion

The incorporation of fuel carbon into the calcined bone samples does introduce the possibility of an old-wood effect in the ${ }^{14} \mathrm{C}$ dates. However, evidence of such a systematic effect is absent in the data shown in Figure 4, as no unexpectedly old dates were observed on calcined bone, and the bone dates correspond well with ${ }^{14} \mathrm{C}$ dates on charcoal, as well as diagnostic ceramics.

\section{CONCLUSIONS}

${ }^{14} \mathrm{C}$ dating calcined bone samples from the Sanctuary of Zeus on Mt. Lykaion was extremely successful and yielded interesting results, both about the method and the site. In terms of methodology, paired dates on calcined bone and organic seeds and charcoal indicate that the calcined bone dating method works well in this context, and there is little surface contamination of bone samples. Concerning archaeological questions, the dates presented here suggest that, despite some mixed surface deposits observed during excavation, much of the sequence in this part of the site is remarkably intact. This is somewhat surprising, given the intense amount of human activity that occurred at the altar over the centuries. It is possible that there was some vertical movement of very small remains at the site, for example, an outlying charcoal date from sample A8. Alternatively, localized cleaning or site maintenance activities may account for some of the discrepancies in the dates. In particular, the hiatus between 1000 and $1300 \mathrm{BCE}$ and the increased variation between dates on different sample types in the lower part of the site may reflect anthropogenic movement of sediments. Further dating studies, as well as analyses of diagnostic ceramics, will certainly address these questions.

Based on the dates presented here, a tentative model for the formation of the altar can be presented. The site was initially used as a ritual space for thysia starting in the Early Mycenaean period. Considerable variation in dates from the lowest archaeological layers might suggest anthropogenic movement of sediments, but the large quantities of Mycenaean pottery sherds indicate a good deal of activity during this period. Between about 1000 and $500 \mathrm{BCE}$, the site was used extremely intensively, with over $40 \mathrm{~cm}$ of anthropogenic sediments deposited at this time. Ceramic and faunal evidence suggests a continuity of ritual behaviors throughout the use of the site. FTIR absorption peaks at $2012 \mathrm{~cm}^{-1}$ indicate that the bones offered to Zeus were either still fully covered in flesh, or were wrapped in fat as described by early texts that discuss thysia. This ritual behavior probably continued beyond the latest date presented here, because samples were not collected from the uppermost levels of the site.

This study provides a unique crossroads between archaeology, ${ }^{14} \mathrm{C}$ research, and classical texts. With an increased understanding of calcined bone dating in this particular context, we hope to date samples from other areas of the altar. Dating the mixed upper layers would help determine an end date for the use of the site. There are also some thin deposits of unburned sediments from the bedrock layers in certain parts of the altar. Faunal remains in these deposits differ drastically to elsewhere in the site, and might represent a different use of the altar during this period. Dating and analyzing these layers could help us understand the development of the ritual use of animals in the region. Finally, an increase in the spatial sampling of the site would help us better understand the formational history of the altar, and if ritual activities were based in different parts of the site through the centuries.

\section{ACKNOWLEDGMENTS}

The Mt. Lykaion Excavation and Survey Project is a collaboration between the University of Arizona, working under the auspices of the American School of Classical Studies at Athens, together with, and under the direction of, the 39th Ephoreia of Prehistoric and Classical Antiquties in Tripolis, Greece, Dr Anna Karapanagiotou, Ephor and Director. Dr David Gilman Romano and Dr Mary 


\section{B M Starkovich et al.}

E Voyatzis are co-directors of the project. Thanks are due to the Mt. Lykaion team and special thanks to Susan Mentzer, Dan Diffendale, and Alexis Belis for assistance in sample collection. Thanks also to Sherry Fox and Eleni Hasaki for logistical support. We would also like to thank the staff of the NSF-AMS laboratory at the University of Arizona in Tucson, in particular Rebecca Watson, Lori Hewett, Marcus Lee, Alex Leonard, Stephanie Castle, Rich Cruz, Todd Lange, and Mitzi de Martino. Thanks to Antoine Zazzo, Katerina Douka, and Phillipa Ascough for chairing the "Advances in Radiocarbon Pretreatment Protocols" session at the 21st International Radiocarbon Conference in Paris, and to Mark McClure for organizing this volume. This paper was improved by thoughtful comments from 2 anonymous reviewers. The Arizona AMS laboratory was partially supported by NSF instrumentation and facilities grant EAR0929458. The research was funded by NSF grant \#1125523. Ongoing support of the Mt. Lykaion excavation is provided by INSTAP, Annette MerleSmith, The Karabots Foundation and by over 40 individual donors.

\section{REFERENCES}

Bronk Ramsey C. 2009. Bayesian analysis of radiocarbon dates. Radiocarbon 51(1):337-60.

De Mulder G, Van Strydonck M, Boudin M, Lerclercq W, Paridaens N, Warmenbol E. 2007. Re-evaluation of the late Bronze Age and early Iron Age chronology of the western Belgian urnfields based on the ${ }^{14} \mathrm{C}$ dating of cremated bones. Radiocarbon 49(2):499-514.

De Mulder G, Van Strydonck M, Boudin M. 2009. The impact of cremated bone dating on the archaeological chronology of the low countries. Radiocarbon 51(2): 579-600.

Ekroth G. 2007. Meat in ancient Greece: sacrificial, sacred or secular? Food \& History 5(1):249-72.

Ekroth G. 2008. Burnt, cooked or raw? Divine and human culinary desires at Greek animal sacrifice. In: Stavrianopoulou E, Michaels A, Ambos C, editors. Transformations in Sacrificial Practices. From Antiquity to Modern Times. Berlin: LIT Verlag. p 87-111.

Ekroth G. 2009. Thighs or tails? The osteological evidence as a source for Greek ritual norms. In: Brulé P, editor. La norme en matière religieuse en Grèce ancienne. Kernos supplément 21, Liège. p 125-51.

Habelitz S, Pascual L, Durán A. 1999. Nitrogen-containing Apatite. Journal of the European Ceramic Society 19:2685-94.

Habelitz S, Pascual L, Durán A. 2001. Transformation of tricalcium phosphate into apatite by ammonia treatment. Journal of Materials Science 36(17):4131-5.

Hüls CM, Erlenkeuser H, Nadeau M-J, Grootes PM, Andersen N. 2010. Experimental study on the origin of cremated bone apatite carbon. Radiocarbon 52(23):587-99.

Kontopoulos K. 1898. Praktika 17-8.

Kourouniotis K. 1904. Excavations at Lykaion. Ephemeris Archaeologike 153-214. In Greek.

Lange T, Barbetti M, Donahue DJ. 2001. Radiocarbon measurements of tree rings from $14 \mathrm{ka}$ Huon pine. $R a-$ diocarbon 43(2A):449-52.

Lanting JN, Brindley AL. 1998. Dating cremated bone: the dawn of a new era. Journal of Irish Archaeology 9: $1-7$.

Lanting JN, Aerts-Bijma AT, van der Plicht J. 2001. Dating of cremated bones. Radiocarbon 43(2A):249-54.

Lebon M, Reiche I, Bahain J-J, Chadefaux C, Moigne AM, Fröhlich F, Sémah F, Schwarcz HP, Falguères C. 2010. New parameters for the characterization of diagenetic alterations and heat-induced changes in fossil bone mineral using Fourier transform infrared spectrometry. Journal of Archaeological Science 37(9): 2265-76.

Mentzer SM. Forthcoming. Appendix 3: The micromorphology of Mt. Lykaion: 2006-2010 field seasons. In: Voyatzis ME, Romano DG. Mt. Lykaion Excavation and Survey Project, Part 1: Upper Sanctuary. Preliminary Report 2004-2010. Hesperia.

Mentzer SM, Voyatzis M, Romano DG. Forthcoming. Micromorphological contributions to the study of ritual behavior at the Ash Altar to Zeus on Mt. Lykaion, Greece. Archaeological and Anthropological Sciences.

Naysmith P, Scott EM, Cook GT, Heinemeier J, van der Plicht J, Van Strydonck M, Bronk Ramsey C, Grootes PM, Freeman SPHT. 2007. A cremated bone intercomparison study. Radiocarbon 49(2):403-8.

Olsen J, Heinemeier J, Bennike P, Krause C, Hornstrup KM, Thrane H. 2008. Characterization and blind testing of radiocarbon dating of cremated bone. Journal of Archaeological Science 35(3):791-800.

Olsen J, Hornstrup KM, Heinemeier J, Bennike P, Thrane H. 2011. Chronology of the Danish Bronze Age based on ${ }^{14} \mathrm{C}$ dating of cremated bone remains. Radiocarbon 53(2):261-75.

Olsen J, Heinemeier J, Hornstrup KM, Bennike P, Thrane H. 2012. 'Old wood' effect in radiocarbon dating of prehistoric cremated bones? Journal of Archaeological Science 40(1):30-4.

Quarta G, Calcagnile L D'Elia M, Maruccio L, Gaballo V, Caramia A. 2012. A combined PIXE-PIGE ap- 


\section{${ }^{14} \mathrm{C}$ Dates from the Sanctuary of Zeus on Mt. Lykaion}

proach for the assessment of the diagenetic state of cremated bones submitted to AMS radiocarbon dating. Nuclear Instruments and Methods in Physics Research B 294:221-5.

Reimer PJ, Baillie MGL, Bard E, Bayliss A, Beck JW, Blackwell PG, Bronk Ramsey C, Buck CE, Burr GS, Edwards RL, Friedrich M, Grootes PM, Guilderson TP, Hajdas I, Heaton TJ, Hogg AG, Hughen KA, Kaiser KF, Kromer B, McCormac FG, Manning SW, Reimer RW, Richards DA, Southon JR, Talamo S, Turney CSM, van der Plicht J, Weyhenmeyer CE. 2009. IntCa109 and Marine09 radiocarbon age calibration curves, 0-50,000 years cal BP. Radiocarbon 51(4): $1111-50$.

Romano DG. 2005. A new topographical and architectural survey of the Sanctuary of Zeus at Mt. Lykaion. In: Østby E, editor. Ancient Arcadia (Papers from the Third International Seminar on Ancient Arcadia, held at the Norwegian Institute at Athens, 7-10, May 2002). Athens: Norwegian Institute of Athens. p 38196.

Romano DG, Voyatzis ME. Forthcoming. Mt. Lykaion Excavation and Survey Project, Part 1: Upper Sanctuary. Preliminary Report 2004-2010. Hesperia.

Sheridan A. 2003. New dates for Scottish Bronze Age cinerary urns: results from the National Museums of Scotland dating cremated bones project. In: Gibson AM, editor. Prehistoric Pottery: People, Pattern and Purpose. BAR International Series 1156. Oxford: Archaeopress. p 206-61.

Shipman P, Foster GF, Schoeninger M. 1984. Burnt bones and teeth: an experimental study of colour, morphology, crystal structure and shrinkage. Journal of Archaeological Science 11(4):307-25.

Slota Jr PJ, Jull AJT, Linick TW, Toolin LJ. 1987. Prep- aration of small samples for ${ }^{14} \mathrm{C}$ accelerator targets by caltalyic reduction of CO. Radiocarbon 29(2):303-6.

Starkovich BM. Forthcoming. Appendix 5: Mt. Lykaion preliminary faunal report. In: Romano DG, Voyatzis ME. Mt. Lykaion Excavation and Survey Project, Part 1: Upper Sanctuary. Preliminary Report 2004-2010. Hesperia.

Stiner MC, Kuhn SL, Weiner S, Bar-Yosef O. 1995. Differential burning, recrystallization, and fragmentation of archaeological bone. Journal of Archaeological Science 22(2):223-37.

Thompson TJU, Gauthier M, Islam M. 2009. The application of a new method of Fourier transform infrared spectroscopy to the analysis of burned bone. Journal of Archaeological Science 36(3):910-14.

Van Strydonck M, Boudin M, Hoefkens M, De Mulder G. 2005. ${ }^{14} \mathrm{C}$-dating of cremated bones, why does it work? Lunula. Archaeologia Protohistorica 16:61-3.

Van Strydonck M, Boudin M, De Mulder G. 2009. ${ }^{14} \mathrm{C}$ dating of cremated bones: the issue of sample contamination. Radiocarbon 51(2):553-68.

Van Strydonck M, Boudin M, De Mulder G. 2010. The carbon origin of structural carbonate in bone apatite of cremated bones. Radiocarbon 52(2-3):578-86.

Weiner S, Bar-Yosef O. 1990. States of preservation of bones from prehistoric sites in the Near East: a survey. Journal of Archaeological Sciences 17(2):187-96.

Wright LE, Schwarcz HP. 1996. Infrared and isotopic evidence for diagenesis of bone apatite at Dos Pilas, Guatemala: palaeodietary implications. Journal of Archaeological Science 23(6):933-44.

Zazzo A, Saliège J-F, Person A, Boucher H. 2009. Radiocarbon dating of calcined bones: Where does the carbon come from? Radiocarbon 51(2):601-11. 